\title{
Genetic Algorithms
}

Genetic algorithms are among the universal optimization methods that allow solving problems of various types (combinatorial, general problems with and without restrictions) and of varying degrees of complexity. Moreover, genetic algorithms are characterized by the possibility of both single-criteria and multi-criteria search in a large space, the landscape of which is nonsmooth [3].

In recent years, the number of works, especially foreign scientists, devoted to the development of the theory of genetic algorithms and questions of their practical use, has increased dramatically. The results of these studies show, in particular, that genetic algorithms can be more widely used when integrated with other methods and technologies. There are works that prove the effectiveness of the integration of genetic algorithms and methods of the theory of fuzziness, as well as neural computing and systems.

\section{Neural networks}

Neural networks are a class of models based on biological analogy with the human brain and designed after passing through the stage of the so-called training on the available data to solve various problems of data analysis. When applying these methods, first of all, the question arises of choosing a specific network architecture (the number of "layers" and the number of "neurons" in each of them).

Data mining is not only used database tools or software. Data mining can be performed with relatively modest database systems and simple tools, including creating your own, or using off-the-shelf software packages. Sophisticated data mining relies on past experience and algorithms defined using existing software and packages, and different specialized tools are associated with various methods.

1. Gavrilova, T.A. Intelligent systems knowledge base / T.A. Gavrilova, V.F. Horoshevskij. - SPb. : PITER, 2001. -382 p.

2. Davydenko, V. Data Mining / V. Davydenko // Software products and systems. - 2007. - № 3(79). - P. 20 31.

3. Korneev, V.V. Database. Intelligent information processing / V.V. Korneev, A.F. Gareev, S.V. Vasyutin. M. : NOLIDZH, 2001. -351 p.

\section{Komlev G.V., Mitrofanova A.S.} Expert systems

Reshetnev Siberian State University of Science and Technology (Russia, Krasnoyarsk)

doi: $10.18411 / \mathrm{lj}-10-2019-28$

idsp: ljournal-10-2019-28

\section{Аннотация}

В статье рассматриваются определение экспертных систем, их структура, классификация, и также технология работы с ними.

Ключевые слова: экспертная система, искусственный интеллект, анализ, синтез.

\section{Abstract}

The article discusses the definition of expert systems, their structure, classification, and also the technology of working with them.

Keywords: expert system, artificial intelligence, analysis, synthesis.

Artificial intelligence, as a scientific discipline, consists of several large currents. One of them is expert systems. 
The methods of artificial intelligence gained wide practical fame precisely during the creation of this special class of programs.

The first such programs appeared in the late 60 s of the last century and were intended to create an artificial "supermind" in some subject area.

In the 60-70s, most expert systems were thought of as some kind of software mechanism that allows you to accumulate the experience of the best expert people, and then advise less experienced users. Classic databases did not fit this role because their query language was not flexible enough to describe the full variety of queries in the real language. In addition, the database query languages did not allow the query to call itself. Within the framework of this approach, the main features of the modern technology of application and construction of expert systems have been formed.

In the modern sense, an expert system is a highly specialized software package that allows you to either make standard decisions very quickly (most often for managing technical objects) or, on the basis of a long dialogue with the user, help in choosing a solution (by evaluating options offered by the user and correcting them taking into account many factors possibly unknown to the user, as well as suggesting your own solutions and their correction in accordance with the goals of the user) [1].

Solving an applied problem with the help of an expert system is always a purposeful iterative process with the goal of developing a solution that is best from some point of view.

The main difference in the work of expert and "non-expert" systems is that the first in each iteration use a new algorithm of actions in the subject area, which differs from the algorithm of the previous step not only in parameters, but also in structure.

The main structural difference of the expert system from all other types of programs is the presence of a knowledge base and, as a result, the ability to learn and self-study. Its specific form strongly depends on the chosen representation model, but in the most general form it will always contain the actual and algorithmic parts [2].

Expert systems can be classified on various grounds: by area of application, by purpose, by performance indicators and others.

The deep model is called, which allows you to reasonably prove your conclusions. Deep knowledge includes abstractions, images, analogies, which reflect the understanding of the structure of the subject area, the purpose and relationship of individual concepts, wellknown laws and theoretical foundations.

Superficial knowledge relates mainly to the external manifestations of the object of study. They allow you to make some assumptions. The conclusions drawn from superficial knowledge are probably as reliable and valuable as the conclusions drawn from deep ones, but they are not substantiated. Superficial knowledge usually refers only to the totality of empirical associations and causal relationships between concepts of the subject area. In principle, almost any model of artificial intelligence is initially born as a surface one, and then, having proved its "usefulness", it gets a solid mathematical foundation, a way of interpreting and explaining the results of the model.

Most expert systems now use superficial knowledge. The introduction of deep representations allows you to create databases of greater power, as deep knowledge is more flexible and adaptive than sufficiently rigid surface. A classic example is medicine, where the in-depth knowledge of experienced doctors allows them to generate various methods of treating the same disease, depending on the condition of the patient, his age, the availability of drugs and other factors.

Deep knowledge is formed as a result of a generalization of the primary concepts of the subject area into some more abstract structures.

The "rigidity" and "softness" of knowledge indicates the ability to receive unambiguous, clear recommendations under given initial conditions, or multiple, vague decisions and recommendations. According to this classification, subject areas themselves can 
be classified as hard and soft. The current trend in the development of expert systems is to move from the widespread use of hard surface models in most problems to soft deep ones.

Expert systems of interpretation and visualization are most often used in economic systems. The fact is that the calculated coefficients used, for example, by the director in the daily management of the enterprise, are difficult not only to analyze, but even simply to keep in mind. The analysis of the values of the coefficients is often not very complicated and consists, for example, in the fact that the value of a certain coefficient should not go beyond a certain framework, provided that the value of another coefficient is in other defined frames, and so several hundred or even thousands of interrelated coefficients .

In this situation, it is extremely important to visualize the data, that is, to visualize. The simplest option is to create charts.

However, if the coefficients are in a certain relationship, it is necessary to use a more complex visualization. Visualization is not always possible. It is important that the explanations are brief (reduce the amount of data held in the head) and contain conclusions about the causes and effects, dependencies, possible solutions and their consequences. To solve the problems of interpretation there is a whole theory of mathematical and statistical data analysis.

Expert diagnostic systems were one of the first to be developed. Together with expert monitoring systems, they solve the problems of continuous diagnostics of technical objects, for example, missiles at the time of launch and during the flight. We can say that such expert systems perform the process of classifying an object to a certain class and detecting the place and type of malfunction. A malfunction is a deviation from the norm. Such an interpretation allows us to consider equipment malfunctions in technical systems, diseases of living organisms, and all kinds of natural anomalies from a single theoretical point of view.

A feature of expert monitoring systems is that it is often necessary to take into account a very large number of parameters that continuously come from hundreds or even thousands of sensors with different types of scales.

In this case, it is often necessary to adhere to a real time scale in order to give an alarm on time.

Expert planning and design systems have much in common with visualization and interpretation systems. For example, such a system, having analyzed the electrical drawing of a new car, can predict the places of possible mechanical damage to the wires during operation. To solve this problem, we will use a special "cognitive" graphics, and methods for enumerating options, and a number of specific methods that experienced design engineers will teach the system. In modern expert systems, the latter is most often performed using the scenario model. If the project is carried out in the field of economics, then instead of drawings we will have a business plan.

Expert forecasting systems logically derive probable consequences from given situations. In a forecasting system, a parametric dynamic model is usually used, in which the parameter values are "adapted" to this situation.

Expert training systems diagnose errors in the study of a discipline using a computer and suggest the right solutions. They accumulate knowledge about the hypothetical "student" and its characteristic mistakes, then in work they are able to diagnose weaknesses in the students' knowledge and find the appropriate means to eliminate them.

In the general case, all knowledge-based systems can be divided into systems that solve the problems of analysis, and systems that solve the problems of synthesis. The main difference between analysis problems and synthesis problems is that if in analysis problems many solutions can be listed and included in the system, then in synthesis problems many solutions are potentially constructed from component solutions or subproblems. The objectives of the analysis are: data interpretation, diagnostics. The tasks of synthesis are: design, planning.

Combined tasks: training, monitoring, forecasting. 
Autonomous expert systems work directly in consultation mode using only for "expert" tasks, the solution of which does not require the involvement of traditional data processing methods.

Hybrid expert systems represent a software package that aggregates standard application software packages and knowledge manipulation tools.

It can be an intellectual add-on over a package of application programs or an integrated environment for solving a complex problem with elements of expert knowledge. Despite the outward attractiveness of the hybrid approach, it should be noted that the development of such systems is an order of magnitude more complex than the development of an autonomous expert system. Docking not just different packages, but different methodologies creates a complex of theoretical and practical difficulties.

Static expert systems are developed in subject areas in which the knowledge base and interpreted data do not change during the solution of a problem. They are stable. For example, troubleshooting a car.

Quasi-dynamic expert systems interpret a situation that changes over a fixed time interval.

Dynamic expert systems work with data changing during the solution of a problem, often in conjunction with object sensors, sometimes in real time with continuous interpretation of incoming data [3]. application.

According to leading experts, in the near future, expert systems will find wide

The fields of application of knowledge-based systems can be grouped into several main classes: medical diagnostics, monitoring and control, fault diagnosis in mechanical and electrical devices, training.

Diagnostic systems are used to establish a connection between disorders of the body and their possible causes. The most famous diagnostic system is MYCIN, which is designed to diagnose and monitor the patient's condition with meningitis and bacterial infections. Its first version was developed at Stanford University in the mid-70s. Currently, this system makes a diagnosis at the level of a specialist. It has an expanded knowledge base, due to which it can be applied in other areas of medicine.

Predictive systems predict possible results or events based on data on the current state of an object. The Wall Street Conquest software system can analyze market conditions and, using statistical methods of algorithms, develop a long-term investment plan for you. It does not belong to the number of knowledge-based systems, since it uses the procedures and algorithms of traditional programming. Although there are as yet no expert systems that can, due to their information on market conditions, help you increase your capital, forecasting systems today can predict the weather, yield and passenger flow. Even on a personal computer, by installing a simple knowledge-based system, you can get a local weather forecast.

Planning systems are designed to achieve specific goals when solving problems with a large number of variables. For the first time in trading practice, Damascus company Informat has provided buyers with 13 workstations installed in the lobby of their office, where free 15minute consultations are held to help customers choose the computer that best suits their needs and budget. In addition, Boeing uses expert systems to design space stations, as well as to identify causes of aircraft engine failures and helicopter repairs. The XCON expert system created by DEC serves to determine or modify the configuration of computer systems such as VAX and in accordance with the requirements of the buyer. DEC is developing a more powerful XSEL system, including the XCON knowledge base, to help customers choose the right computing system for their configuration. Unlike XCON, XSEL is interactive.

Interpretative systems have the ability to obtain certain conclusions based on observation results. The PROSPECTOR system, one of the most famous systems of the interpretive type, combines the knowledge of nine experts. Using a combination of nine 
examination methods, the system managed to detect ore deposits worth a million dollars, and none of the nine experts suggested the presence of these deposits. Another interpretive system is HASP / SIAP. It determines the location and types of ships in the Pacific Ocean according to acoustic tracking systems.

Knowledge-based systems can be used as intelligent control systems and make decisions by analyzing data from multiple sources. Such systems already operate at nuclear power plants, control air traffic and exercise medical control. They can also be useful in regulating the financial activities of the enterprise and assist in the development of decisions in critical situations.

In this area, knowledge-based systems are indispensable both in the repair of mechanical and electrical machines and in the elimination of malfunctions and errors in the hardware and software of computers.

Knowledge-based systems can be integrated into computer-based training systems. The system receives information about the activity of an object (for example, a student) and analyzes its behavior. The knowledge base changes in accordance with the behavior of the object. An example of this training is a computer game, the complexity of which increases as the skill level of the player increases. One of the most interesting training expert systems is the EURISCO system developed by D. Lenat, which uses simple heuristics. This system was tested in the game T. Treveveller, simulating military operations. The essence of the game is to determine the composition of the flotilla that can defeat in an unchanging set of rules. The EURISCO system included small ships capable of carrying out a quick attack and one very small high-speed vessel in the flotilla and constantly won for three years, despite the fact that in an effort to prevent this, the rules of the game were changed every year.

Most expert systems include knowledge, the content of which can be attributed to several types simultaneously. For example, a training system may also have the knowledge to perform diagnostics and planning. It determines the abilities of the student in the main areas of the course, and then, taking into account the received data, draws up a curriculum. The control system can be used for monitoring, diagnostics, forecasting and planning. The home security system can monitor the environment, recognize events (for example, a window opens), issue a forecast (a burglar intends to enter the house) and draw up an action plan (call the police).

$$
\text { *** }
$$

1. Nilson, N. Principles of Artificial Intelligence / N. Nilson. - M.: Radio i svyaz', 2014. - 373 p.

2. Uoterman, D. Building expert systems / ed. F. Hejes-Rot, D. Uoterman, D. Lenat. - M.: Mir, 2013. - 441 p.

3. Shapiro, S.I. Human Thinking and Computer Processing / S.I. Shapiro. - M.: Sovetskoe radio, 2017. - 288 p.

\section{Mitrofanova A.S., Komlev G.V. Neural network data processing technologies}

Reshetnev Siberian State University of Science and Technology (Russia, Krasnoyarsk)

doi: $10.18411 /$ lj-10-2019-29

idsp: ljournal-10-2019-29

\section{Аннотация}

Изложены математические и алгоритмические аспекты функционирования искусственных нейронных сетей. Рассмотрены парадигмы обучения нейронных сетей, архитектура и нейросетевые модели.

Ключевые слова: нейросетевые технологии, архитектура нейросетей, нейросетевая модель, обработка информации 